\title{
Retinal photography using panchromatic and orthochromatic films
}

\author{
A. GRAANDIJK AND A. L. AAN DE KERK \\ Eye Hospital, Rotterdam, The Netherlands
}

Much has been written about fundus photography and the efforts to obtain better results 1 the use of different cameras and filters, but little mention has been made of the choice of film. Drews (1957) showed that the chromatic abberrations of the optical systems of the patient and camera could be largely eliminated by use of a monochromatic light sourco. Behrendt and Wilson (r965), and Behrendt and Duane (rg66) introduced the concept $\vec{g}$ retinal spectral reflectance photography, using various interference filters and comparifg the negatives obtained at a specified wavelength.

Our purpose is to show that for black-and-white photography similar results can obtained by using orthochromatic and panchromatic films.

\section{Materials and methods}

A modified Zeiss fundus camera is used in the photographic department of the Rotterdam E Hospital, with the same diaphragm No. 7. A Nikon motor transport camera is employed for black and-white photography. All patients are photographed with colour film as well as with black-an $\vec{\phi}$. white orthochromatic and panchromatic film. The advantage of not using interference filters twofold:

(1) A great amount of flash is absorbed ( 96 per cent., according to some authors) and lost for phot graphy. This indicates the need for fast films, resulting in coarse-grained pictures. The alternatioc is the use of a very high intensity flash power unit. The one we use gives an energy of 840 watts $/ \mathrm{see}$., which is found to be too low in intensity to use interference filters successfully without also using hig ASA-rating film.

(2) Interference filters are expensive and cumbersome to build into the Zeiss fundus camera.

There are several advantages with the graphic films: a high standard of enlargement is obtaine without coarse graining as well as a high resolving power and contrast; greater detail can be observf because of the absence of graining; and graphic films are inexpensive.

The films we used were Agfa Copex Ortho and Agfa Copex Pan. The spectral sensitivity curve్ are shown in Fig. I (opposite). The Ortho film has a peak in the $585 \mathrm{~nm}$. range and the Pan the $63^{\circ} \mathrm{nm}$. range. The sensitivity is about Io ASA for the Ortho and a little faster for the Pan fil

If other wavelengths are required it is possible to use special sensitized spectroscopic film with spectral range of 250 to $770 \mathrm{~nm}$.

The great advantage of the graphic films is their high resolving power-Ortho 350 lines per mrat and Pan 500 lines per $\mathrm{mm}$. In the large group of patients photographed it was endeavoured $\overrightarrow{\mathrm{d}}$ photograph the same place at one sitting, and two exposures were taken with each film, wi位 different intensities, allowing for the small tolerance latitude. 
All films were developed in a diluted solution of Promicrol developer ( $1: 2)$ at a constant temperature. This is contrary to the normal, low-contrast, panchromatic film which has to be force developed.

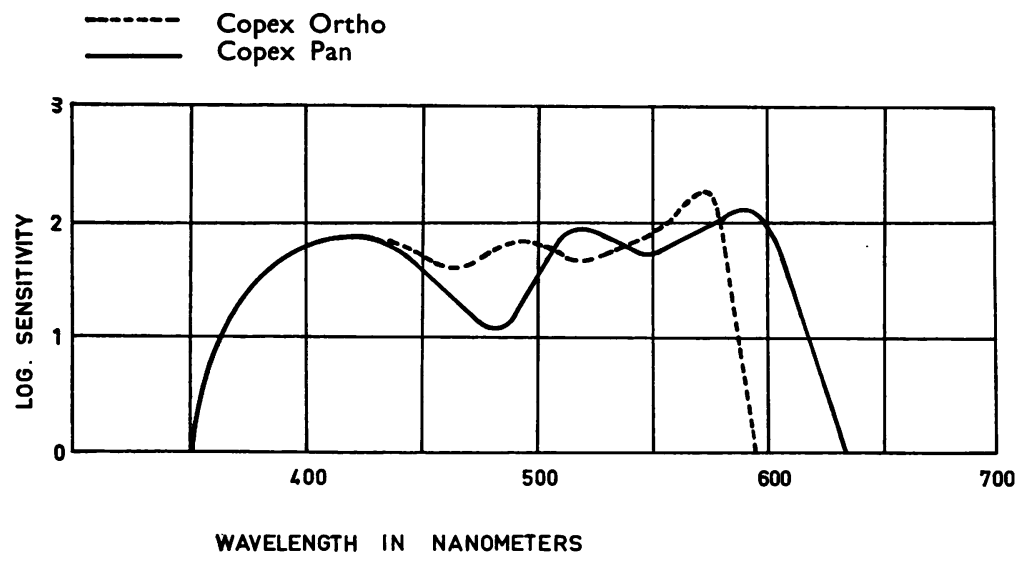

F I G. I Spectral sensitivity curves of Copex Ortho and Copex Pan films

\section{Results}

The photographic material presented (Figs 2-7) has enabled the following comparison to be made:

Panchromatic (Copex Pan $630 \mathrm{~nm}$.)

Reflections: few

More veiling through cataract due to "false light"

Optic disc : highly reflectant; colour: white

Pigmentations: great detail, very fine pigmentations seen

Vessels: arteriovenous difference more pronounced, but vessel detail on the whole is less

Nerve fibre layer: not seen
Orthochromatic (Copex Ortho $585 \mathrm{~nm}$.)

Reflections from retina and vessels: more numerous

Hardly any veiling

Optic disc: darker, more detail, the smaller vessels are clearly discernible; colour: grey

Pigmentations: Less detail, finer pigmentary changes not seen

Vessels : more detailed, especially the smaller ones

Nerve fibre layer: quite often seen

\section{Discussion}

In accordance with Behrendt and Wilson (1965) and Behrendt and Duane (1966) is the finding that more detail of the retinal structures is obtained, in their case using interference filters, in our case using films of different spectral sensitivity.

They implied a further penetration by light of different wavelengths. Whether this is a true deeper penetration or whether it is due to differences in contrast is not so easily solved, and further experimental work is required.

The results suggest that it is reasonable to use orthochromatic film, since greater detail is obtained; only the pigmentary changes are shown in greater detail on panchromatic film. 

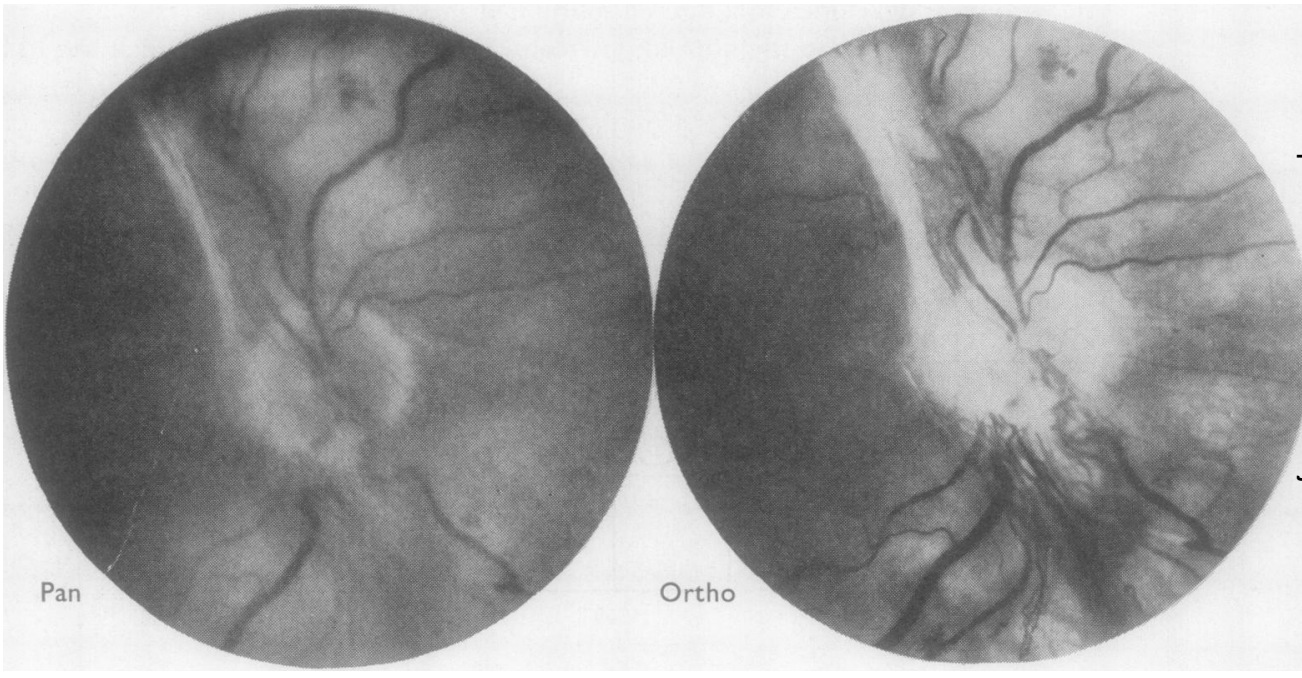

FIG. 2 Proliferation in diabetic retinopathy

PAN : Ill-defined optic disc with poor vessel detail. Proliferation not clearly defined. Overall hazy aspect d产 to veiling by cataract

овтно: Clearly-defined optic disc and proliferation. Vessel detail excellent. No veiling caused by cataract
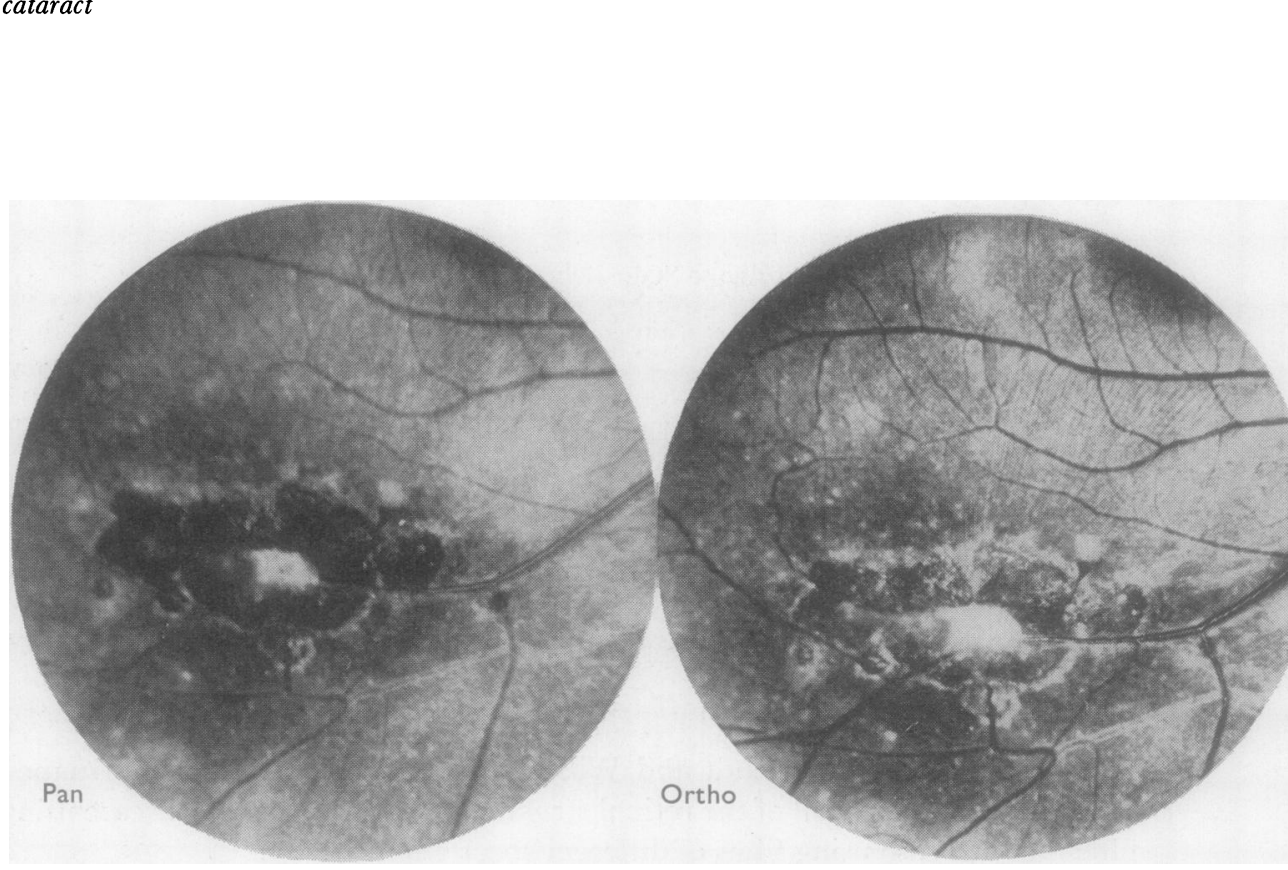

FIG. 3 Choroiditis scar

PAN : Black scar very distinct; occluded vessel not clearly seen; vessel detail on the whole poor

о т тно: Scar less black but shows more detail; occluded vessel clearly seen; vessel detail good; neurofibril laye just discernible 


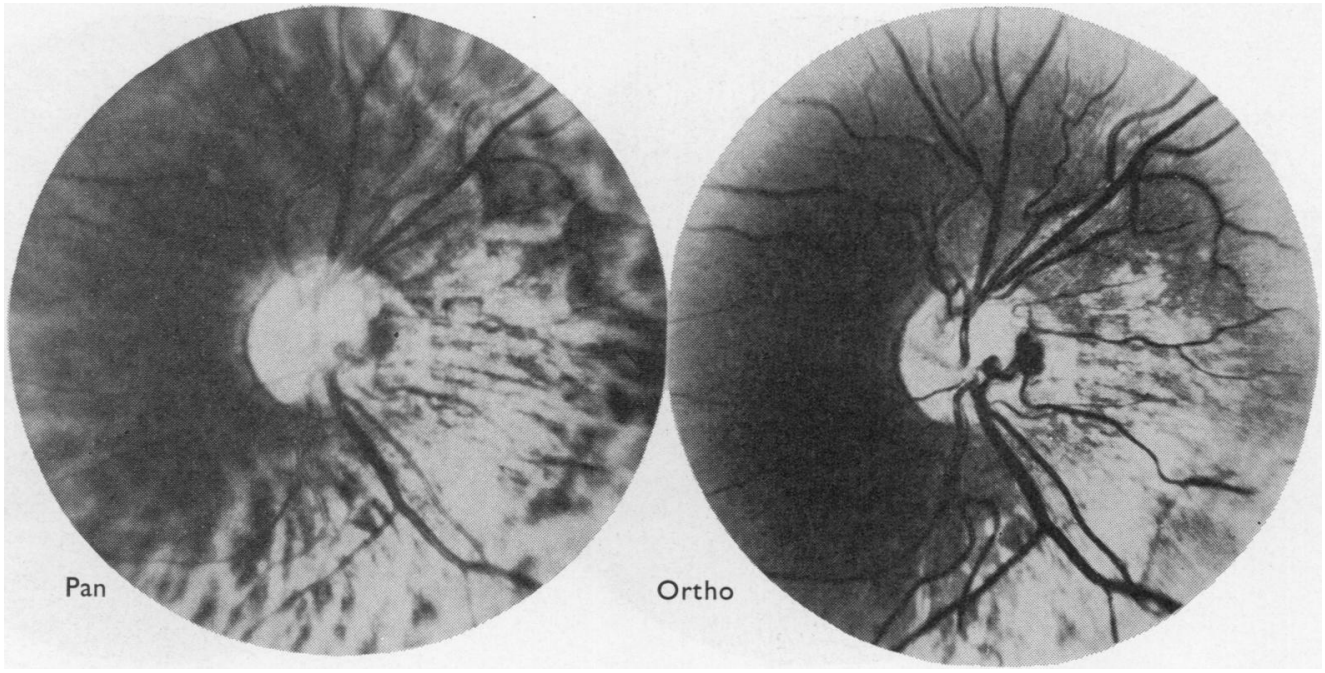

FIG. 4 Persistent hyaloid artery and cilioretinal vessels

PAN : Vague detail of hyaloid remnant, cilio-retinal vessels hardly distinguishable; vessel detail on the whole poor O Rтно: Clearly-defined optic disc, the persistent hyaloid artery and cilioretinal vessels are easily seen
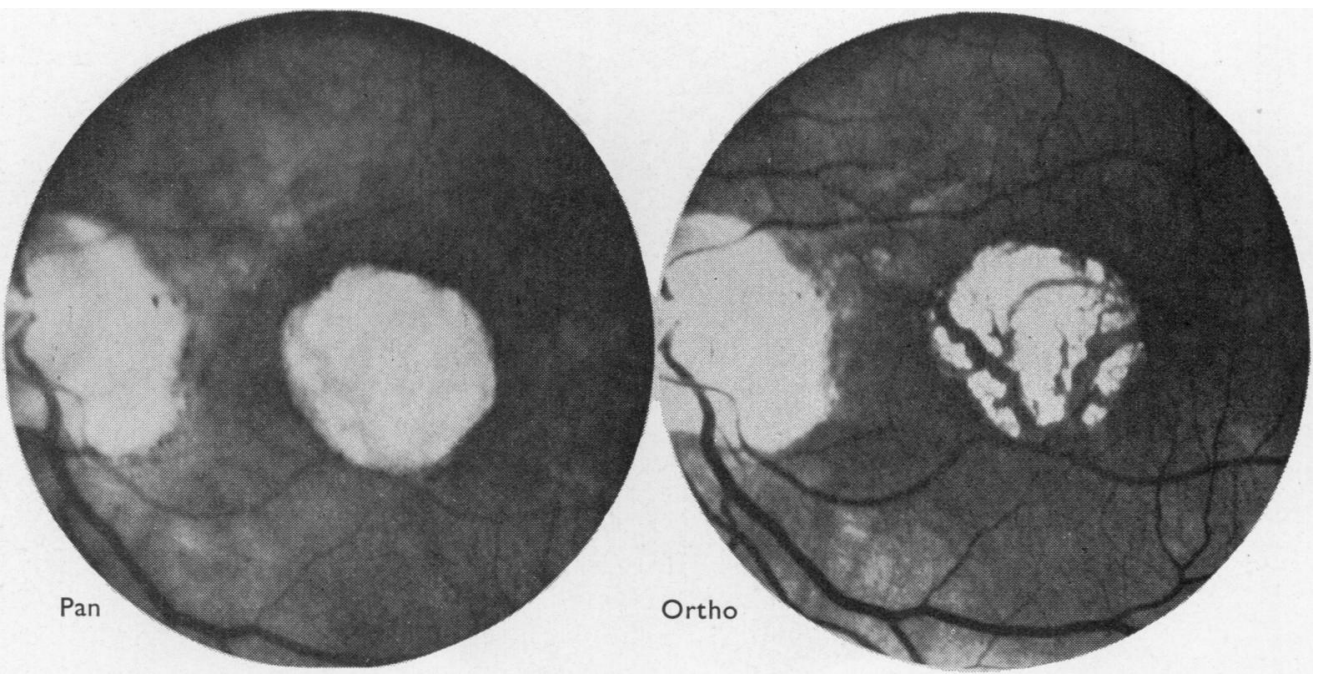

FIG. 5 Central choroidal sclerosis

PAN : White central choroidal sclerosis with vague detail of choroidal vessels

овтно: Clearly-defined central area, with clear choroidal vessels 

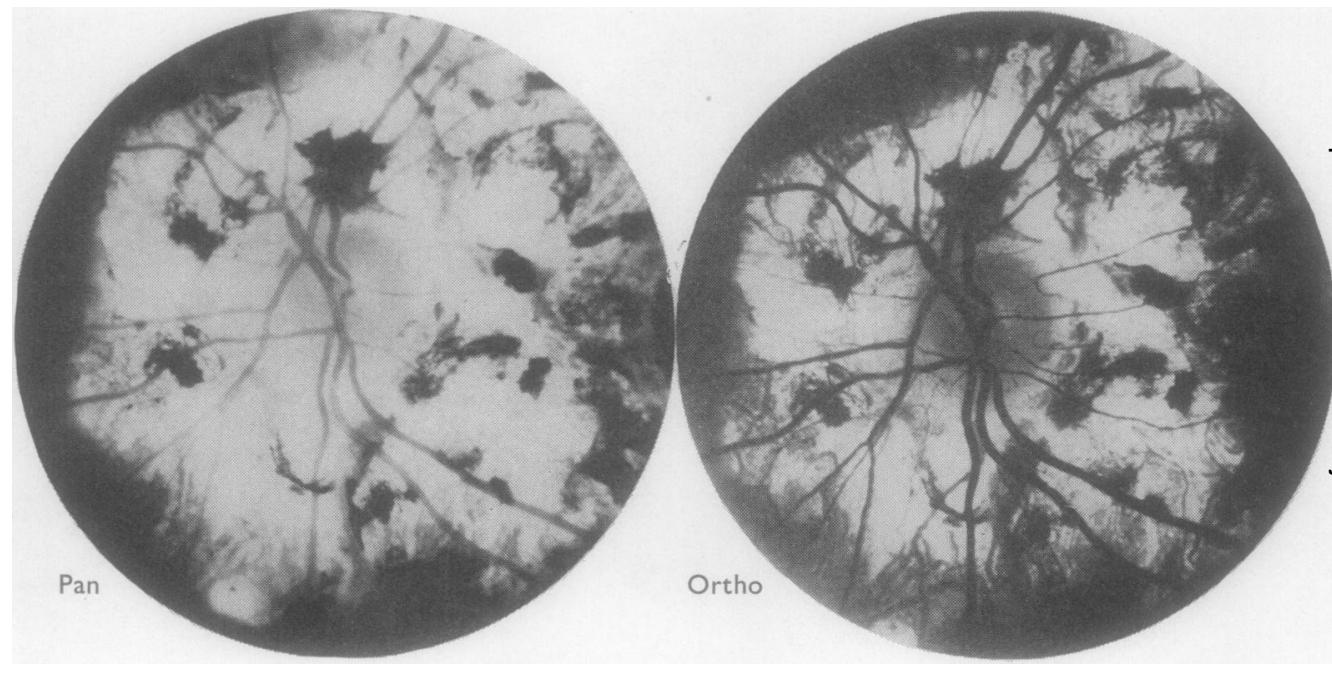

FIG. 6 Peripapillary atrophy

PAN : Optic disc white and ill-defined; papillary vessels not seen; pigmentations in atrophic area pronounced

orTHo: Optic disc grey, with clearly-defined papillary vasculature; retinal vessels clearly discernible pigmentations less defined

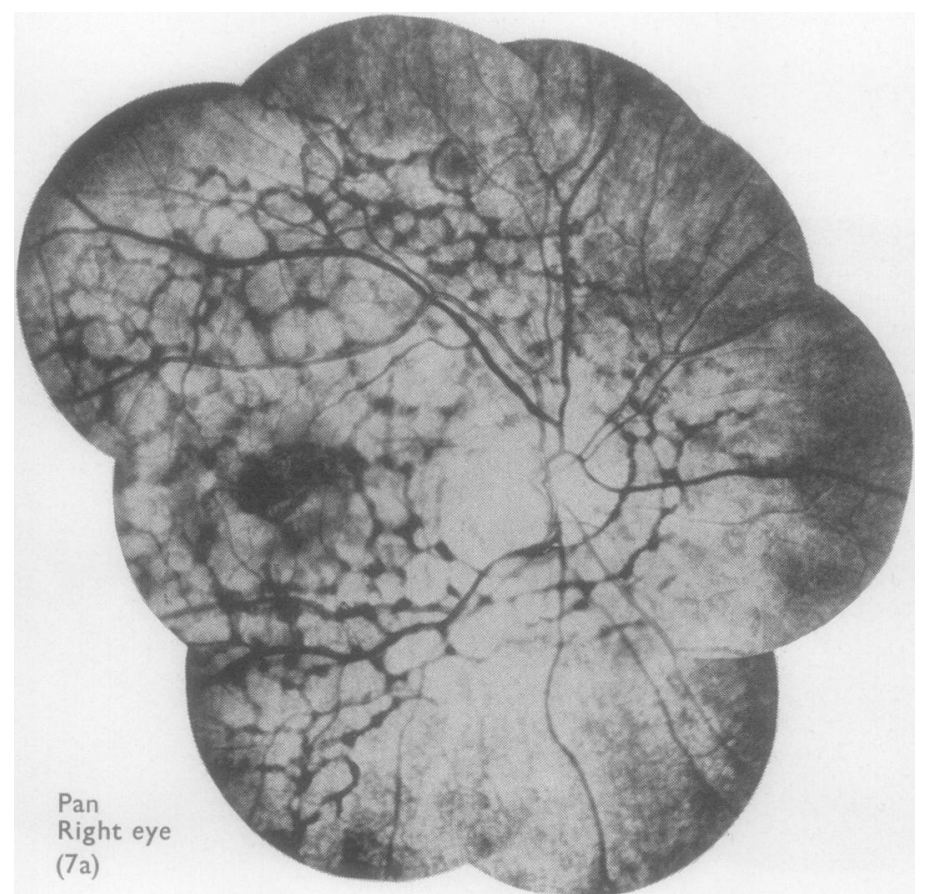

FIG. 7 Reticular dystrophy of retinal pigment epithelium (Deutman, unpublished)

PAN : Optic disc white; vessel detail less clear; reticular pigmented network clearly seen

O R тно: Optic disc clearly defined; vessel detail more pronounced; reticular network barely discernible (opposite page) 


\section{Summary}

A photographic series is presented to illustrate the different results obtained with panchromatic and orthochromatic graphic film.

On the whole far more detail of the optic disc and vessels is obtained with orthochromatic film, but with pigmented lesions, often changes in the deeper retinal layers and pigment epithelium, better results are obtained with panchromatic film.

It would therefore seem advisable to use orthochromatic graphic film for routine fundus photography, rather than the normal panchromatic film usually used.

It should be pointed out that the film materials used are primarily slow, high-contrast $35 \mathrm{~mm}$. films designed for micro-filming and are available only in roo-ft. lengths.

We are indebted to Dr. A. F. Deutman for permission to use a photograph of his patient with reticular dystrophy of the retinal pigment epithelium.

\section{References}

BEHRENDT, T., and DUANE, T. D. (1966) Arch. Ophthal. (Chicago), 75, 375 and Wilson, L. A. (1965) Amer. 7. Ophthal., 59, 1079

DEUTMAN, A. F. "Reticular dystrophy of the retinal pigment epithelium" (to be published) Drews, R. C. (1957) Amer. 7. Ophthal., 44, 356

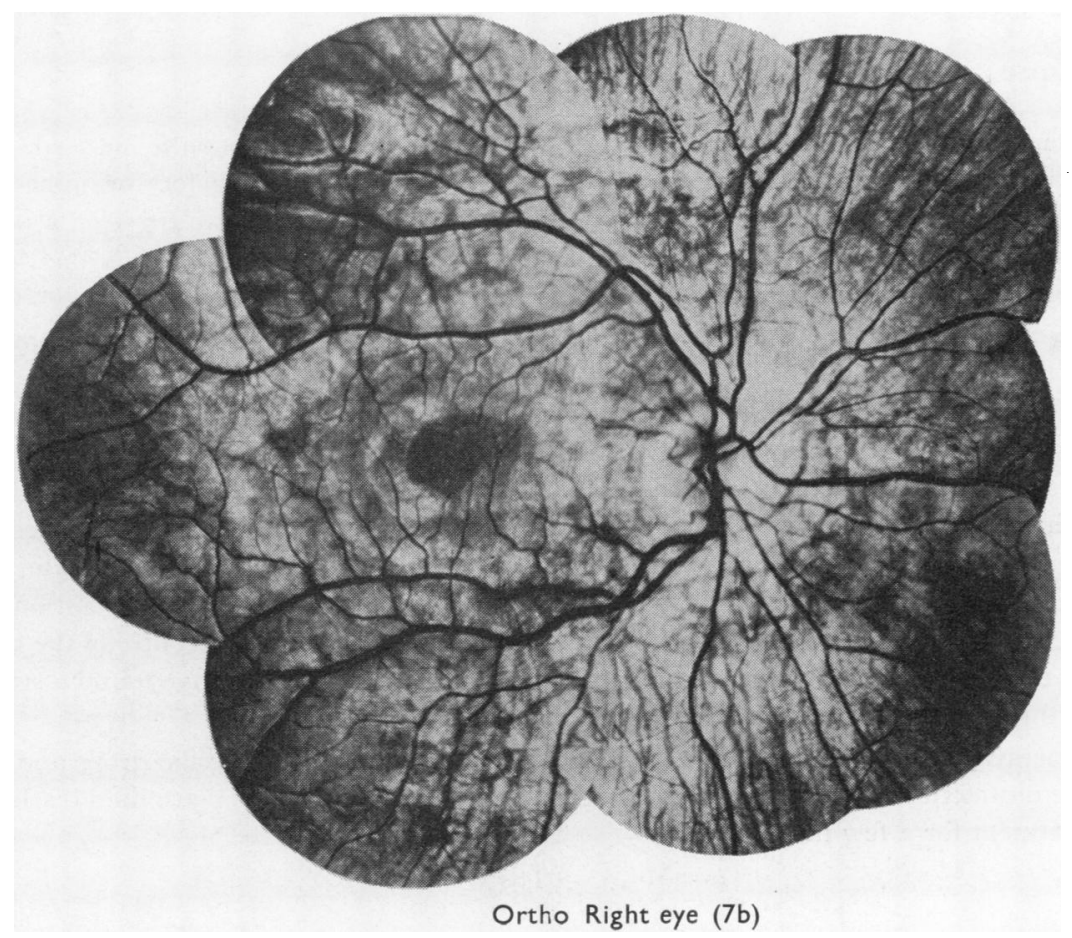

